\section{C ummary}

In this article, an opinion is presented on the calibration of dissolution rate apparatuses using the USP method. It is shown that significant within- and between-apparatus variability can exist for certain tablet formulations, even though these apparatuses meet the USP calibration criteria. The implications of variability from "calibrated" dissolution rate apparatuses (DRAs) are discussed. In light of these data, it is suggested that the workload required to complete the present USP calibration procedure be reduced. In addition, the establishment of product specific laboratory standards is recommended to track dissolution assay performance over an extended period. It is hoped that this article will help to spark scientific discussion regarding improved methods for the calibration of dissolution rate apparatuses. in each dissolution flask. In theory, calibration of a DRA could be accomplished solely by the measurement of key mechanical and alignment parameters (rotational speed accuracy, flask centering, verticality of paddle/basket shafts, wobble, and vibration) with appropriate performance limits for each measurement. The USP calibration procedure goes one step further by requiring the measurement of dissolution rate for salicylic acid and prednisone tablets, which represent nondisintegrating and disintegrating dosage forms, respectively. To fully calibrate a DRA, one performs dissolution rate tests for each calibrator tablet using the 50 and $100 \mathrm{rpm}$ paddle and basket for a total of 8 tests. The apparatus is considered calibrated if all individual tablets of each run fall within the range provided by the USP with the specific lot of tablets. Having completed this significant

\title{
Calibration Of Disso
Rate Apparatuses: A User's Perspective
}

\section{Introduction}

The purpose of calibrating an analytical instrument is to ensure that measurements made with that instrument are accurate and precise within specified performance limits. The key to calibration is the availability of suitable standards such that the performance of the instrument can be unambiguously assessed against the specified limits. For an HPLC system, we seek to verify that the detector response is linear over a given concentration range with acceptable noise characteristics. For this evaluation, any material of known purity can be used such that accurate concentrations can be prepared. Instruments which meet the calibration criteria can be expected to give identical results on a given set of samples.

Calibration of a DRA is conducted to ensure the proper mechanical functioning and physical alignment of the unit such that uniform and reproducible hydrodynamics are produced task (requiring at least 2-3 person days for an experienced analyst to perform) it is not without some anxiety that the analyst compares the individual tablet data to the range of dissolution rates provided by the USP with the specific lot of tablets. If all of the tablets fall within the range, then the apparatus is considered calibrated. If one or more tablets are out of range, then what? The answer to this question is probably specific to each laboratory, but it is highly likely that the majority of laboratories rerun that specific test and if the range is met then the apparatus is considered calibrated. While it is outside the scope of this article to suggest alternate calibration procedures, it is appropriate to discuss the pros and cons of the current procedure.

It is worth noting that there are tangible benefits and logical arguments for running the USP calibrator tablets. After making the

(continued on page four)

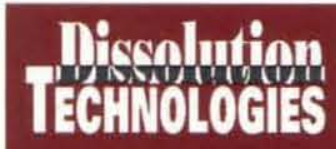

John W.

Skoug, Ph.D. 


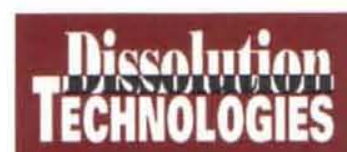

Figure 1: Effect of

dissolution rate

apparatus on

dissolution rate.

Error bars

represent the

standard deviation

obtained by

averaging the

mean dissolution

result at 30

minutes for

between 3 and 16

six-tablet

dissolution runs

collected for the

same tablet lot.

The data were

collected over an

18 month period

\section{Effect of Apparatus on Dissolution Rate}

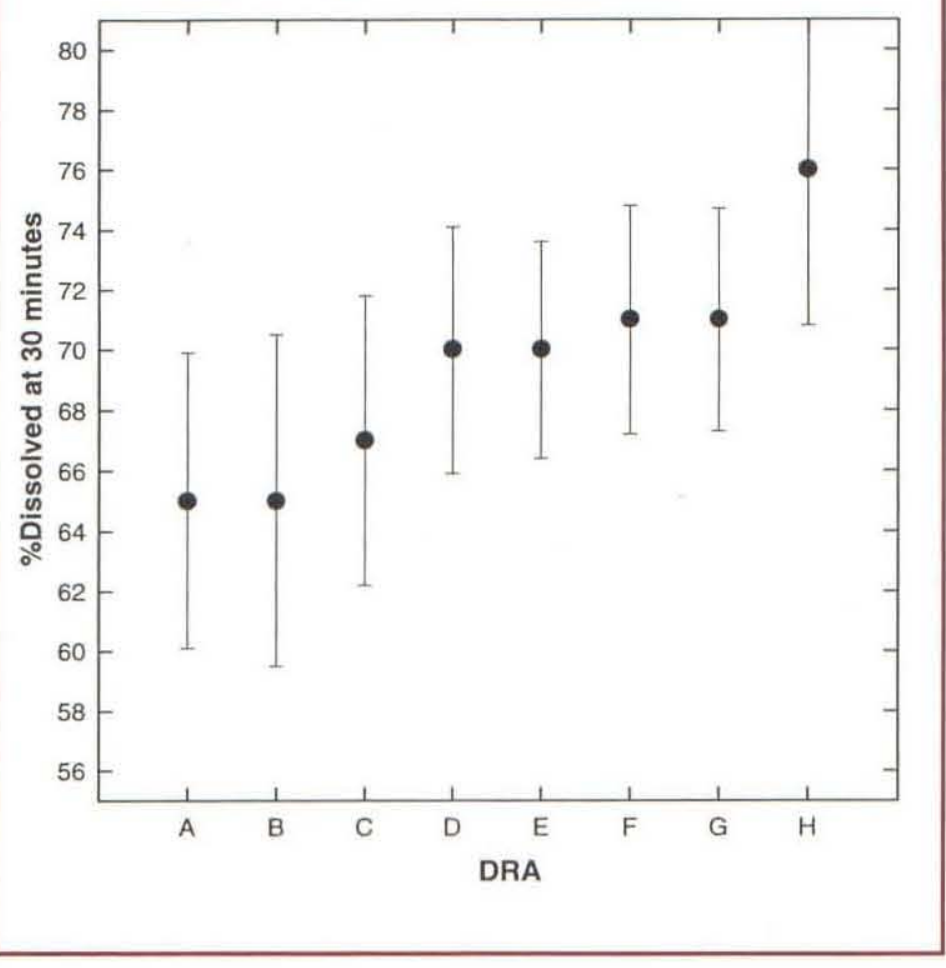

brated," can give a false sense of security with regard to the accuracy of the dissolution results that will be obtained for a given pharmaceutical dosage form. For example, Figures 1 and 2 show the between- and within- apparatus variability that is obtained for a product being developed at The Upjohn Company. For all dissolution results, the USP paddle apparatus at 75 rpm is used with $900 \mathrm{ml}$ of $0.1 \mathrm{~N} \mathrm{HCl}$ as the dissolution medium. Figure 1 shows the mean dissolution result at 30 minutes for the same tablet lot obtained using several DRA's, all of which meet the USP calibration criteria. The data clearly show that there is a significant effect of dissolution rate apparatus on the mean dissolution result. Figure 2 shows that the within apparatus "position effect," the systematic variation in dissolution results with DRA flask position, is also a significant effect.

(continued from page 3 )

necessary mechanical and physical alignment measurements, it is appropriate to perform a dissolution run using tablets for which there exists a significant body of data. The benefit of this exercise is not only to provide assurance that the alignment measurements were conducted correctly, but also to ensure that analyst technique is incorporated into the final result. Because of the relative simplicity of the dissolution assays for each calibrator tablet they are a useful and convenient tool to train new analysts in the nuances of conducting dissolution tests. Meeting the dissolution limits, therefore, provides a simple and convenient way to standardize the conduct of dissolution tests in laboratories around the world.

On the other hand, meeting the dissolution limits supplied with each lot of calibrator tablets, thus labelling a given DRA as "cali-
The magnitude of this so-called position effect varies from DRA to DRA, but is remarkably reproducible across many runs on a given DRA. The specific cause(s) for the variability illustrated in Figures 1 and 2 is not yet understood, however, it has been determined that these results are related to the DRA being used and not to the analyst running the method. Experience shows that these types of apparatus effects are most likely to be observed for drugs of low aqueous solubility when formulated into immediate release tablets that require greater than 60 minutes to achieve complete dissolution.

In light of these data, it is fairly obvious that the USP calibrator tablets do not represent standards that will universally 


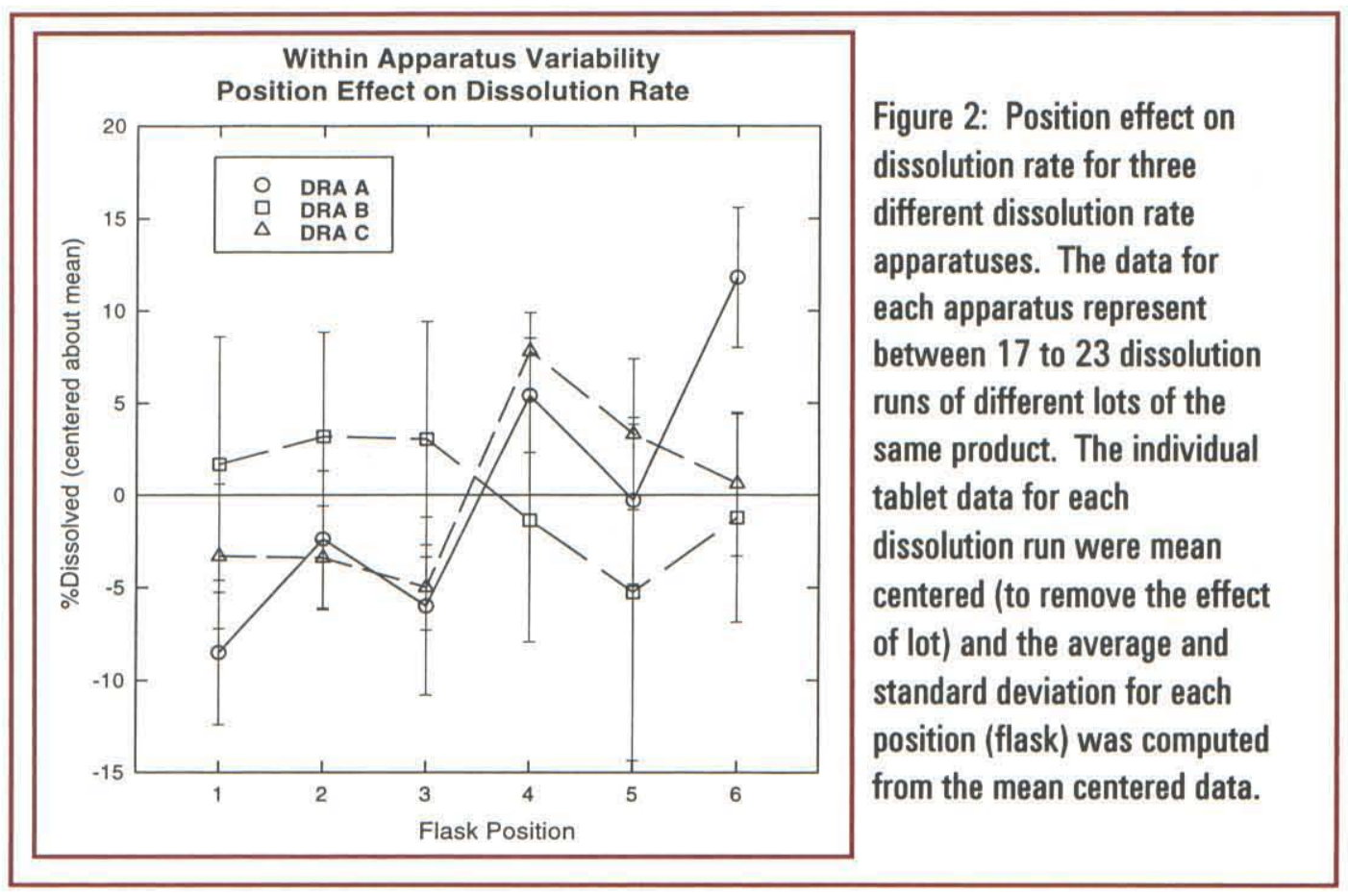

guarantee equivalent DRA performance for all pharmaceutical products (perhaps it would be unrealistic to expect this of any dosage form). If this argument is accepted, then what is the implication with regard to calibration of DRA's using the current USP procedure? To be sure, the answer to this question is complicated, but two proposals representing short- and long-term approaches are presented to begin the discussion.

A short-term solution is to re-evaluate the current USP calibration procedure in light of both the benefits and the deficiencies noted above. For example, based on the data in Figures 1 and 2, it is appropriate to ask whether the considerable resource required for calibration by the USP procedure is worthwhile. It seems illogical to run eight dissolution tests to complete a calibration procedure that does not assure equivalent results across apparatuses for all dosage forms. If the calibration procedure is viewed as a means to assure that gross apparatus differences do not occur, then the workload could easily be reduced by one-half without compromising the result of the calibration in any way. For example, running only the four most sensitive tests $(50 \mathrm{rpm}$ paddle and 50 rpm basket) for each product seems logical. Alternately, since the $50 \mathrm{rpm}$ basket is almost never used in practice, these 2 tests could be eliminated.

A longer-term solution is to reevalute the DRA calibration procedure in its entirety. While this topic is outside the scope of this article, it is worthwhile to raise the question of whether better alternatives to the current calibrator tablets do in fact exist. The issue of improved strategies for DRA calibration might well be a fertile topic for discussion at a future scientific meeting.

In closing, the deficiencies in the current dissolution calibration procedure highlight the importance of establishing and maintaining a laboratory standard program. Establishment of a laboratory standard involves selecting a representative tablet lot for each product and then periodically testing that lot. Data obtained from the laboratory standard are important because they provide a convenient way to monitor assay performance over an extended period of time, thus providing a measure of assay ruggedness. Analyzed appropriately, these data are more useful for tracking the performance of specific assays and DRA's in a quality control setting, than the semi-annual calibration data obtained from the USP calibrator tablets.

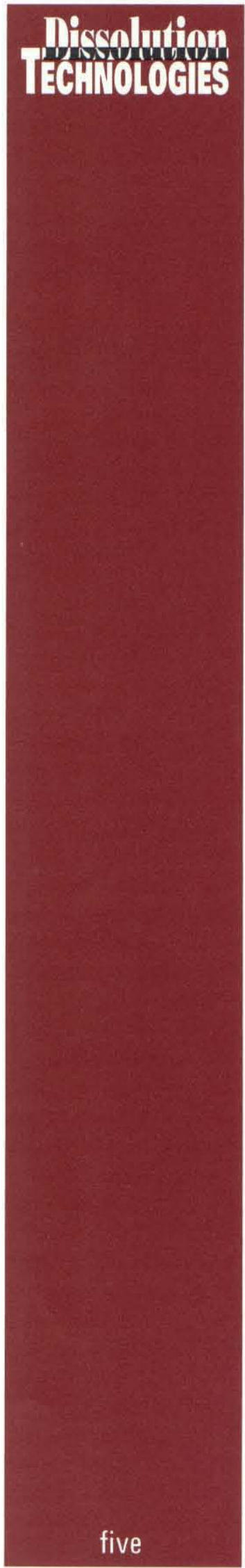

